\title{
Scattering of surface plasmon polaritons by one-dimensional inhomogeneities
}

\author{
A. Yu. Nikitin,,${ }^{1,2, *}$ F. López-Tejeira, ${ }^{1}$ and L. Martín-Moreno ${ }^{1, \dagger}$ \\ ${ }^{1}$ Departamento de Física de la Materia Condensada-ICMA, Universidad de Zaragoza, E-50009 Zaragoza, Spain \\ ${ }^{2}$ Theoretical Physics Department, A. Ya. Usikov Institute for Radiophysics and Electronics, Ukrainian Academy of Sciences, \\ 12 Acad. Proskura Str., 61085 Kharkov, Ukraine
}

(Received 9 September 2006; revised manuscript received 9 November 2006; published 30 January 2007)

\begin{abstract}
The scattering of surface plasmons polaritons by a one-dimensional defect of the surface is theoretically studied by means of both Rayleigh and modal expansions. The defects considered are either relief perturbations or variations in the permittivity of the metal. The dependence of transmission, reflection, and out-of-plane scattering on parameters defining the defect is presented. We find that the radiated energy is forwardly directed (with respect to the surface plasmon propagation) in the case of an impedance defect. However, for relief defects, the radiated energy may be directed into the backward or forward (or both) direction, depending on the defect width.
\end{abstract}

DOI: $10.1103 /$ PhysRevB.75.035129

\section{INTRODUCTION}

The study of electromagnetic excitations localized on metal-dielectric interfaces, or surface plasmon polaritons (SPP's), has become of essential importance due to its potentiality for the implementation of subwavelength photonic circuits. ${ }^{1,2}$ As a result of such a study, phenomena like total suppression of reflection, ${ }^{3}$ transformation of polarization, ${ }^{4-6}$ enhanced light transmission, ${ }^{7,8}$ formation of band gaps ${ }^{9-12}$ and other interesting properties of plasmonic crystals have been discovered. Lately, a great deal of attention has been devoted to the creation of optical elements for SPP's, ${ }^{13-17}$ as well as to the efficient coupling of light into and out of SPP's. These latter problems require a precise knowledge of the scattering coefficients of the dispersion centers (i.e., deviations from a flat metal-dielectric interface) placed on the path of SPP's. However, while many theoretical works have studied the problem of SPP scattering by rough surfaces, scattering from simple geometries is not so well known. This was undoubtedly due to the previous lack of reproducible experimental data, which are now available thanks to recent advances in the controlled patterning of metal surfaces.

From the theoretical side, the calculation of electromagnetic (EM) fields on a metal surface in the optical regime is a well-defined but difficult problem. Although the macroscopic Maxwell equations govern very accurately the interaction of the EM fields with the solid, their solution is difficult due to the different ranges of length scales involved (system size, wavelength, skin depth, etc.). Several techniques have been applied to this problem, each of them with their advantages and drawbacks. The Greens's dyadic technique $^{18-20}$ or the discrete dipole approximation ${ }^{21-23}$ are virtually exact methods which suffer from the large (quite often prohibitive) numerical cost involved with the inversion of huge matrices and the need to calculate cumbersome Sommerfeld integrals. On the other hand, the mode-matching technique is computationally much simpler, ${ }^{24}$ but it can only be directly applied to the case of indentations in a metal film (and not to protrusions). ${ }^{25,26}$ Additionally, it requires the use of a surface impedance boundary conditions (SIBC), which is only applicable when the metallic dielectric constant $\epsilon$ satisfies $|\epsilon| \gg 1$. An alternative approach based on the Rayleigh approximation (which is valid for small scatterers) has
PACS number(s): 73.20.Mf, 78.67.-n, 41.20.Jb

been also extensively applied in the context of EM scattering by rough surfaces. ${ }^{27}$ Even within this approximation, the calculation of the scattering coefficients requires solving a difficult integral equation. ${ }^{28-30}$ Several works have rendered this integral equation into a more manageable form by means of an additional approximation, which assigns a geometrydependent "local impedance" to the surface relief (see, for instance, Refs. 31-33). To our knowledge, all works have concentrated on scatterers with translational symmetry in one direction, onto which the SPP impinges at normal incidence, except for the case of a circularly symmetric defect, considered in Ref. 30 by using the reduced Rayleigh equation.

In this work, we use the Rayleigh approximation combined with the SIBC at the metal-vacuum interface, systematically taking into account the geometry of the surface. Our first result is an integral equation governing the SPP scattering by arbitrary perturbations, which can be either spatial variations in metal permittivity or in surface profile. This integral equation is as simple as the one defined by a "local impedance," $31-33$ but is far more accurate, as shown by comparisons with results obtained using the mode-matching technique. Due to its simplicity, the method developed provides a clear physical description of SPP scattering effects. As a first application, here we concentrate on the scattering properties of a single inhomogeneity, as a function of shape and geometrical parameters defining the defect.

\section{RAYLEIGH EXPANSION APPROXIMATION}

\section{A. Fields representation and boundary conditions}

Consider a monochromatic surface plasmon with frequency $\omega$, impinging along a metal-vacuum interface at normal incidence (with in-plane wave vector $k_{p}$ ) onto an inhomogeneity region; see Fig. 1. In keeping with other works on scattering of SPP's, we consider that the metal is lossless. From a physicist's point of view, we expect this to be valid in the systems we have in mind, as far as the dimension of the inhomogeneity in the direction of SPP propagation is smaller than the absorption length. More mathematically, SPP scattering channels are well defined in a lossless metal, and current conservation provides a strong (although not definitive) test for the theory. Nevertheless, the proposed method could, 


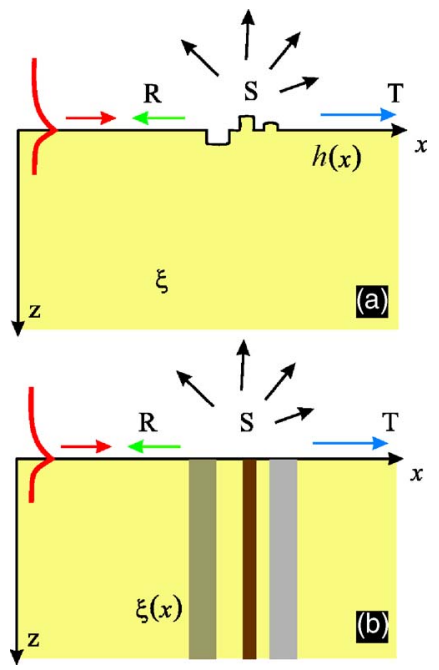

FIG. 1. (Color online) Schematic illustration of the studied system: SPP scattering at the inhomogeneity formed by either (a) the perturbation of the interface profile $h(x)$ or (b) the surface impedance of the metal $\xi(x)$.

mutatis mutandis, accommodate for absorption. In this case, instead of scattering coefficients, the outcome of the calculation would be spatial EM field distributions which could be used, for instance, to analyze scanning near-field optical microscope or leakage radiation experiments.

The considered inhomogeneities can be due to variations either in the surface impedance or in the surface profile. Although the integral equations governing scattering will have the same functional form for both cases, the derivations are slightly different. Let us concentrate first on the latter case, which is somewhat more involved. Suppose that the metal has a dielectric constant $\epsilon$ (and correspondingly a surface impedance $\xi=1 / \sqrt{\epsilon})$ and that the surface relief profile has the functional form

$$
z=h(x), \quad h(x)=\int d k h(k) \exp (i k x)
$$

Assuming that both the variation of the surface relief and its derivative are small $\left(|h| \ll \lambda\right.$ and $\left.\left|\partial_{x} h\right| \ll 1\right)$, we may represent the field over the surface in the form of a Rayleigh expansion. ${ }^{34}$ The nonzero EM field components in the vacuum half-space can be expanded in terms of the incident SPP plus scattered field as

$$
\begin{aligned}
\left\{\begin{array}{c}
E_{x}(x, z) \\
E_{z}(x, z) \\
H_{y}(x, z)
\end{array}\right\}= & \left\{\begin{array}{c}
-k_{z p} / g \\
-k_{p} / g \\
1
\end{array}\right\} \exp \left[i\left(k_{p} x-k_{z p} z\right)\right] \\
& +\int d k\left\{\begin{array}{l}
E_{x}(k) \\
E_{z}(k) \\
H_{y}(k)
\end{array}\right\} \exp \left[i\left(k x-k_{z} z\right)\right],
\end{aligned}
$$

where $g=\omega / c=2 \pi / \lambda, k_{z}=\sqrt{g^{2}-k^{2}}$, and $k_{z p}=\sqrt{g^{2}-k_{p}^{2}}=-\xi g$. The branch of the square root should be chosen such that $\operatorname{Im}\left(k_{z}\right) \geqslant 0$, in order to satisfy the radiation condition. Notice that, within the SIBC, the SPP dispersion relation is $k_{p}$ $=g\left(1-\xi^{2}\right)^{1 / 2}$, which approximates the exact SPP dispersion relation $k_{p}=g[\epsilon /(1+\epsilon)]^{1 / 2}$ at $|\epsilon| \gg 1$.

Within the SIBC, ${ }^{35}$ the EM fields should satisfy

$$
\mathbf{E}_{t}(x, z)=\xi \mathbf{H}_{t}(x, z) \times \mathbf{n}(x), \quad \text { at } z=h(x),
$$

where $\mathbf{n}=\left(n_{x}, 0, n_{z}\right)$ is the unitary vector normal to the surface (directed into the metal half-space) and subscript $t$ corresponds to the tangential components of the fields.

Notice that the SIBC assumes that the radius of curvature of the surface is much larger than the skin depth. However, as the comparison with results obtained by the modal expansion method will show, the SIBC still represents accurately scattering by defects where this condition is not fulfilled at a small number of points (as occurs for rectangular and triangular indentations).

\section{B. Integral equation for the scattered field amplitudes}

Expressing the tangential component of the electric field as

$$
\mathbf{E}_{t}(x, z)=\mathbf{E}(x, z)-\mathbf{n}(x)[\mathbf{E}(x, z) \cdot \mathbf{n}(x)],
$$

the $x$ component of Eq. (3) gives

$$
E_{x}(x, z) n_{z}(x)-E_{z}(x, z) n_{x}(x)=\xi H_{y}(x, z), \quad \text { at } z=h(x) .
$$

Substituting the fields from Eq. (2) into Eqs. (5) and using Maxwell equations $E_{x}=-(l / g)(\partial / \partial z) H_{y}$ and $E_{z}=(l / g)$ $\times(\partial / \partial x) H_{y}$ provides an integral equation for the amplitudes of the scattered fields-for instance, for the magnetic component $H_{y}(k)$. A much more manageable equation can be obtained for smooth surface inhomogeneities by expanding the boundary condition over the small parameters $\left|\partial_{x} h\right|$, $|h| / \lambda \ll 1$. As the surface normal vector has the form

$$
\mathbf{n}=\frac{\mathbf{e}_{z}-\mathbf{e}_{x} \partial_{x} h}{\sqrt{1+\left(\partial_{x} h\right)^{2}}}
$$

the expansion of these vector components up to second-order terms gives

$$
\begin{gathered}
n_{z}=1-\frac{1}{2}\left(\partial_{x} h\right)^{2}+O\left(\left|\partial_{x} h\right|^{4}\right), \\
n_{x}=-\partial_{x} h+O\left(\left|\partial_{x} h\right|^{3}\right) .
\end{gathered}
$$

Following an analogous procedure with the exponents $\exp \left(-i k_{z p} h\right)$ and $\exp \left(-i k_{z} h\right)$ appearing in the boundary condition (but in the parameter $|h| / \lambda$ ), after some straightforward algebra we finally obtain an expanded integral equation, in which as many expansion terms (in powers of $\partial_{x} h$ and $|h| / \lambda)$ as necessary should be retained in order to satisfy energy conservation up to a required accuracy. However, as will be shown later, just considering up to the terms linear in $\partial_{x} h,|h| / \lambda$ already provides accurate energy conservation. Moreover, we will show that this even occurs for rectangular or triangular defects which are, in principle, unfavorable cases as the shape slopes are not small everywhere. From the physical point of view this means that small-scale spatial components of the fields do not contribute essentially to the 
scattering. It is convenient to define the dimensionless wavevector components $q=k / g$ (so that $q_{p}=k_{p} / g=\sqrt{1-\xi^{2}}$ ) and $q_{z}=k_{z} / g$ and the dimensionless Fourier amplitude of the relief defect $\eta(q)=i g^{2} h(k)$. Additionally, the renormalized field amplitudes $r(q)$ are defined by

$$
r(q)=g H_{y}(k) G(q)^{-1}, \quad G(q)=1 /\left(\xi+q_{z}\right),
$$

where $G(q)$ is the Green's function corresponding to the unperturbed SPP.

Then we find that SPP scattering is governed by

$$
r(q)+\int d q^{\prime} U\left(q, q^{\prime}\right) G\left(q^{\prime}\right) r\left(q^{\prime}\right)=-U\left(q, q_{p}\right) .
$$

In this equation $U\left(q, q^{\prime}\right)$ is the scattering potential which, in general, can be expressed as a series expansion in the Fourier image of the defect profile $\eta(q)$ as

$$
U\left(q, q^{\prime}\right)=U_{1}\left(q, q^{\prime}\right)+U_{2}\left(q, q^{\prime}\right)+\cdots, \quad U_{n}\left(q, q^{\prime}\right) \sim \eta^{n} .
$$

Up to first order in $\left|\partial_{x} h\right|,|h| / \lambda$, the explicit expression for the potential is ${ }^{36}$

$$
U\left(q, q^{\prime}\right)=\left[q^{\prime}\left(q-q^{\prime}\right)-q_{z}^{\prime} G\left(q^{\prime}\right)^{-1}\right] \eta\left(q-q^{\prime}\right) .
$$

We have to keep in mind that, within the "local impedance" approximation, the SPP scattering is also governed by Eq. (9), but in this case, the scattering potential is $U_{\text {local }}\left(q, q^{\prime}\right)=\eta\left(q-q^{\prime}\right)(\epsilon-1) / \epsilon \approx \eta\left(q-q^{\prime}\right)$. This different functional form is not irrelevant: as we will show later, the fact that $U\left(q_{p}, q_{p}\right)=0$ (property not shared by $U_{\text {local }}$ ) has important consequences on the scattering of SPP by surface reliefs. In order to ascertain which is the best approximation, subsequent sections present comparisons between results obtained with the modal expansion and both potentials $U$ and $U_{\text {local }}$. Let us anticipate that the comparison favors the scattering potential $U\left(q, q^{\prime}\right)$ defined by Eq. (11).

Another point favoring $U\left(q, q^{\prime}\right)$ against $U_{\text {local }}\left(q, q^{\prime}\right)$ is to consider the perfect conductor limit $(\epsilon \rightarrow-\infty)$, where the SIBC must transform to $(\partial / \partial n) H_{y}(x, z)=0$, evaluated at $z$ $=h(x)$. In this limit, while Eq. (3) transforms correctly, the use of "local impedance" leads [by using Eqs. (5) and (25) of Ref. 31] to the boundary condition $(\partial / \partial z) H_{y}(x, z)$ $=g^{2} h(x) H_{y}(x, z)$, evaluated at $z=0$. The root of the problem seems to be that the series expansion in $h(x)$ [Eq. (2.23) in Ref. 31] diverges as $\epsilon \rightarrow \infty$ (note, for instance, that the second-order term is inversely proportional to the skin depth, $d \sim 1 / \sqrt{-\epsilon}$.

To complete this section, let us point out that the previously outlined formalism can be generalized to the case of defects due to impedance inhomogeneities. In this case, the metal surface is flat, but the surface impedance becomes a function of the $x$ coordinate,

$$
\xi(x)=\xi+\widetilde{\xi}(x)=\xi+\int d k \widetilde{\xi}(k) \exp (i k x) .
$$

After applying the SIBC, which in this case reads $E_{x}(x, z$ $=0)=\xi(x) H_{y}(x, z=0)$, we find that SPP scattering is also con- trolled by Eq. (9). The difference is that now the scattering potential is

$$
U\left(q, q^{\prime}\right)=\eta\left(q-q^{\prime}\right)
$$

where $\eta(q)=g \widetilde{\xi}(k)$ is the dimensionless Fourier amplitude of the modulation defect.

\section{Transmission, reflection, and out-of-plane scattering}

Once the coefficients $r(q)$ are obtained, the integrals in Eqs. (2) define the EM field everywhere in vacuum. However, in order to find the scattering coefficients, only the asymptotic values at large distances from the scattering center are needed. It is then convenient to prolong the subintegral functions into the complex plane, taking into account the presence of poles. While the renormalized Fourier image of the field, $r(q)$, is not expected to present poles, the Green's function $G(q)$ definitely does. In order to retain its causal character, an infinitesimally small damping should be included as $\xi \rightarrow i \operatorname{Im}(\xi)+0$. As a result, the magnetic field at the surface $z=0$ may be written in the following form:

$$
\begin{gathered}
H_{y}(x \rightarrow \infty, 0)=(1+\tau) \exp \left(i k_{p} x\right), \\
H_{y}(x \rightarrow-\infty, 0)=\exp \left(i k_{p} x\right)+\rho \exp \left(-i k_{p} x\right),
\end{gathered}
$$

where

$$
\tau=\frac{2 \pi i \xi}{q_{p}} r\left(q_{p}\right), \quad \rho=\frac{2 \pi i \xi}{q_{p}} r\left(-q_{p}\right) .
$$

The energy flux scattered out of the metal-vacuum interface is computed by integrating the Poynting vector over a rectangular parallelepiped defined by the plane $z=0$ and walls placed in the far-field parallel to the planes $O x, O y$, and $O z$. Then, taking into account that the power per unit length of the incoming SPP is $q_{p} /(4|\xi|)$, the energy conservation law has the following form:

$$
1-S-T-R=0,
$$

where the reflection $R$, transmission $T$, and out-of-plane $S$ scattering coefficients are

$$
\begin{gathered}
R=|\rho|^{2}, \quad T=|1+\tau|^{2}, \\
S=\frac{4 \pi|\xi|}{q_{p}} \int_{|q|<1} d q q_{z}|G(q) r(q)|^{2} .
\end{gathered}
$$

The total out-of-plane scattered current can then be written in the following form:

$$
\begin{gathered}
S=\int_{-\pi / 2}^{\pi / 2} d \theta D(\theta), \\
D(\theta)=\frac{4 \pi|\xi|}{q_{p}} \cos ^{2} \theta|r[q(\theta)] G[q(\theta)]|^{2} \\
=\frac{4 \pi|\xi| \cos ^{2} \theta}{q_{p}\left(|\xi|^{2}+\cos ^{2} \theta\right)}|r[q(\theta)]|^{2},
\end{gathered}
$$

where $D(\theta)$ is the differential reflection coefficient (DRC), 

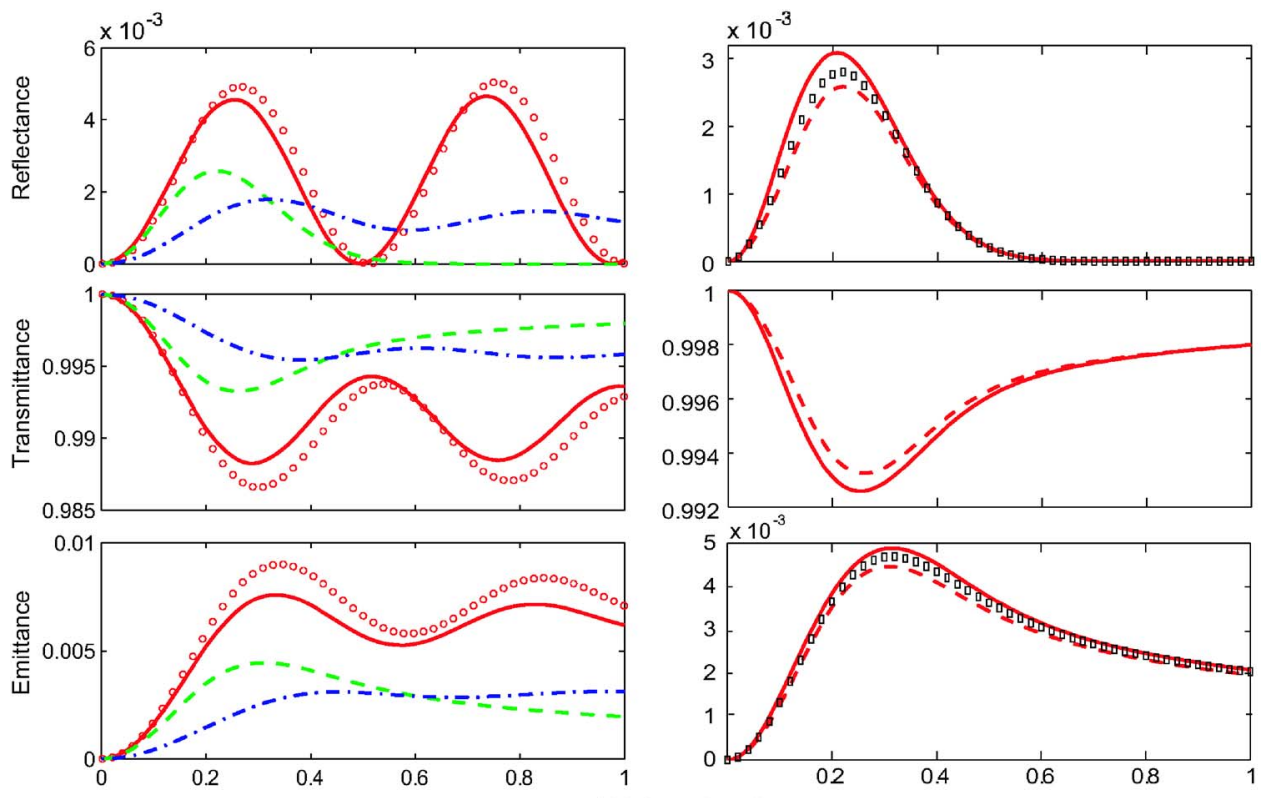

$0.992 \times 10^{-3}$

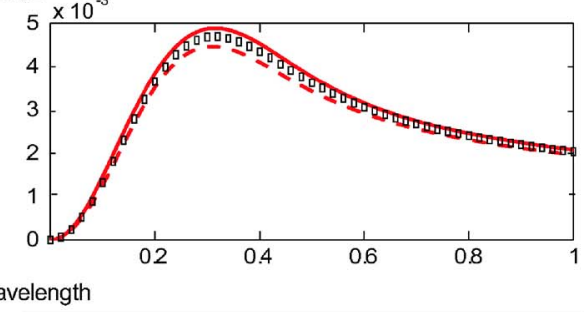

width / wavelength
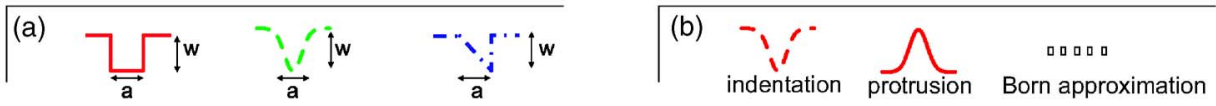

FIG. 2. (Color online) The dependence of the transmittance $T$, reflectance $R$, and emittance $S$ upon the dimensional width of the defect of different shapes in the silver surface (at $600 \mathrm{~nm}$ ). The amplitude is $w / \lambda=0.02$. In (a) solid, dashed, and dash-dotted curves correspond to the rectangular, Gaussian, and triangular shapes of indentation. Round markers correspond to the calculations using mode expansion. In (b) the dashed (solid) curve corresponds to a Gaussian indentation (protrusion); by the rectangular markers the Born approximation is presented. which provides the angular dependence of the radiated energy. In this expression the angle $\theta$ is defined from the normal, while $q(\theta)=\sin \theta$ and $q_{z}(\theta)=\cos \theta$.

To summarize this section, the set of equations (9), (16), and (17) defines the scattering coefficients for an SPP impinging normally into an arbitrary set of perturbations (with an axis of translational symmetry) in a flat metal surface within the Rayleigh + SIBC approximations. Such perturbations can be either variations in the surface relief (either indentations or protrusions) or variations in the surface impedance.

\section{Perturbative approach: Qualitative description of the results}

In some cases, it is useful to estimate the solution of Eq. (9) using a perturbative approach. Taking into account the representation of the scattering potential in Eq. (10), we seek the solution of Eq. (9) in the form of a series expansion in $\eta$ (Ref. 38):

$$
r(q)=r_{1}(q)+r_{2}(q)+\cdots, \quad r_{n}(q) \sim \eta^{n} .
$$

The first-order Born approximation (FOBA) gives us

$$
r_{1}(q)=-U_{1}\left(q, q_{p}\right) \sim \eta\left(q-q_{p}\right) .
$$

The second-order term is

$$
r_{2}(q)=-U_{2}\left(q, q_{p}\right)+\int d q^{\prime} U_{1}\left(q, q^{\prime}\right) G\left(q^{\prime}\right) U_{1}\left(q^{\prime}, q_{p}\right) .
$$

As usual, the FOBA describes the scattering of the incident wave with in-plane wave vector $q_{p}$ into a plane wave with the wave vector $q$, the momentum difference being provided by a single interaction with the defect. Therefore the scattering amplitude for this process is proportional to $\eta\left(q-q_{p}\right)$. This explains the structure of the transmission coefficient. As Eq. (16) shows, $T$ has contributions from both the incident SPP (with unit amplitude) and from the scattering of the incident plasmon "into itself" [the term proportional to $\eta(0)$ ]. Analogously, in the FOBA the amplitude of the reflection is proportional to $\eta\left(-2 q_{p}\right)$ and represents the scattering of the incoming SPP into the backwardly propagating one (having the wave vector $-q_{p}$ ).

\section{SPP SCATTERING BY A SINGLE DEFECT}

In this section we consider the dependence of scattering properties by single one-dimensional (1D) defects of different shapes. The defect width is $a$ and the defect depth is $w$ (see Fig. 2). Notice that, within the chosen coordinate system, indentations are characterized by $w>0$, while protrusions have $w<0$. The calculation is performed by solving numerically the integral equation (9), after applying an appropriate discretization in $q$ space. As the results are in good agreement with those obtained within first-order Born approximation, analytic expressions for the scattering coefficients are provided in several cases.

\section{A. Scattering coefficients}

Figure 2 renders the results of numerical calculations for $R, T$, and $S$ in different instances. In (a) the comparison of these quantities for single indentations of different shapes is presented. In the case of a rectangular shape, $R$ behaves periodically with respect to $a / \lambda$, while in the case of a Gaussian shape $R$ possesses only one maximum. Analogously, the transmittance presents oscillations in $a / \lambda$, in contrast with the single maximum that appears for the case of Gaussian 

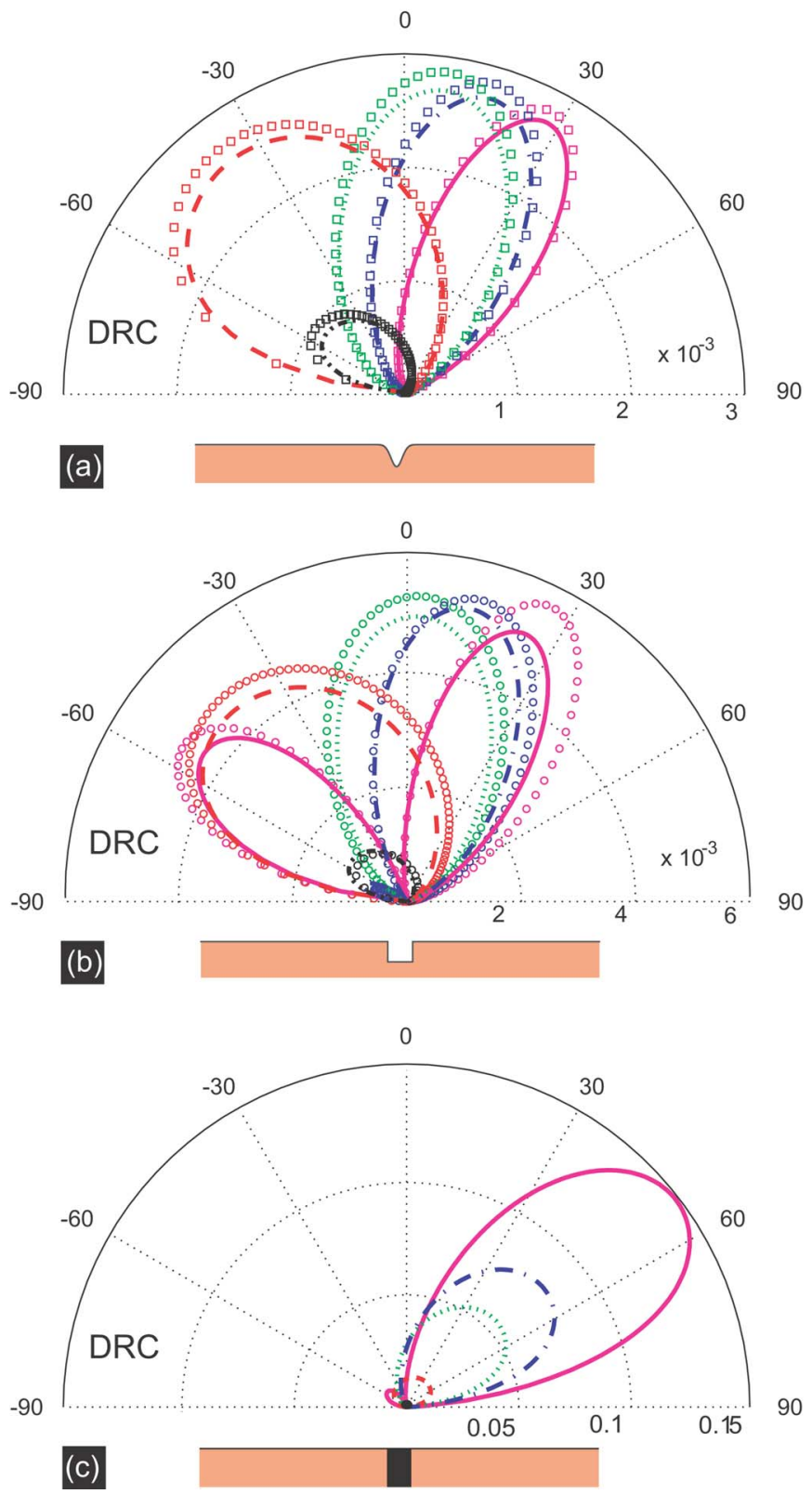

FIG. 3. (Color online) DRC, $D(\theta)$, for SPP scattering along a silver surface $(\lambda=600 \mathrm{~nm})$, for different defect types and widths. (a) Gaussian indentation, (b) rectangular indentation, and (c) impedance step (the metal is considered ideal inside the defect). Dashdouble-dotted (black), dashed (red), dotted (green), dash-dotted (blue), solid (magenta) lines correspond to defect widths $a / \lambda=0.1$, $0.25,0.5,0.6$, and 0.8 , respectively. The depth of the indentation is $w / \lambda=0.02$. Squares in (a) correspond to calculations within the first-order Born approximation. Round markers in (b) correspond to calculations using modal expansion.

shape. In the same figure we present also the calculations for the triangular shape. These results show that even shallow defects present very rich shape and spectral dependences.

Figure 2(b) shows the scattering coefficients for both indentations and protrusions of Gaussian shape. As can be seen from this figure, the scattering properties of indentations and protrusions are very similar for shallow defects. Nevertheless, for the considered parameters, protrusions present a slightly larger cross section, resulting in larger values for both $R$ and $S$ and smaller ones for $T$. We stress that, although the integral equations were derived assuming that the surface was smooth, energy conservation was fulfilled with an accuracy better than $1 \%$ of the minimum value of $R, T$, and $S$, even for sharp defects. Additionally, we have checked that retaining second-order terms in $\eta(q)$ in the scattering potential leave the results virtually unaltered. Moreover, solving the integral equation with the "exact" (not expanded) righthand side does not produce any significant variation in the calculated scattering coefficients.

In order to further validate the above-mentioned approximations, leading to the results presented in Fig. 2, additional calculations were carried out with the modal expansion technique. ${ }^{26}$ While also using the SIBC, this technique is applicable for indentations of any depth, going beyond the Rayleigh expansion. The comparison is presented in Fig. 2(a), for the case of a rectangular indentation. As can be seen, the agreement is very good, the difference being attributed mostly to the fact that, in the modal expansion, ideal metal boundary conditions were used for the vertical "walls" of the rectangular indentation. We note in passing that using the "local impedance" scattering potential gives very different results: the values of $T$ and $S$ differ by more than an order of magnitude from the ones presented in Figs. 2 and 3 (we performed the calculations with the potential presented in Ref. 33 for the same set of parameters).

We find that the FOBA provides an accurate description of the behavior of the calculated scattering coefficients. This is illustrated in Fig. 2(b), where the FOBA results for the Gaussian defect are compared with the full solution of Eq. (9). Notice that the FOBA predicts the same reflectance for both protrusions and indentations, since $R \sim w^{2}$. Further analysis shows that the second-order approximation already accounts for the small differences between the scattering coefficients of indentations and protrusions found in Fig. 2(b). This occurs because, in second-order approximation, $R \sim \mid w$ $+\left.w^{2} \psi(a)\right|^{2}$, where the complex function $\psi(a)$ depends upon both shape and longitudinal size of the defect.

Besides, the FOBA enables us to find analytic expressions for the reflectance of SPP's by a single defect quite easily, in terms of $\left|\eta\left(-2 q_{p}\right)\right|^{2}$. For instance, for a rectangular or a Gaussian defect of width $a$, we obtain

$$
\eta(q)^{\text {rect }}=i \Delta \frac{\sin (q \widetilde{a})}{\pi q}, \quad \eta(q)^{\text {Gauss }}=\frac{i \widetilde{a} \Delta}{2 \sqrt{\pi}} e^{-q^{2} \tilde{a}^{2} / 4},
$$

where $\widetilde{a}=a \pi / \lambda$ and $\Delta=g w$ for relief defects (for impedance defects $\Delta$ corresponds to the maximum value of $|\widetilde{\xi}|)$. Therefore, the reflectance of a single relief defect can be expressed as

$$
\begin{gathered}
R^{\text {Gauss }}=4 \pi|\xi|^{2} q_{p}^{2} \Delta^{2} \widetilde{a}^{2} e^{-2 q_{p}^{2} \tilde{a}^{2},} \\
R^{\text {rect }}=16|\xi|^{2} q_{p}^{2} \Delta^{2} \widetilde{a}^{2} \operatorname{sinc}^{2}\left(2 q_{p} \widetilde{a}\right) .
\end{gathered}
$$

Thus, for rectangular defects $R$ behaves periodically as a function of $a / \lambda$, possessing minima at $a / \lambda=n / 2 q_{p}, n$ $=1,2, \ldots$, while the reflectance for defects of Gaussian shape presents only one maximum at $a / \lambda=1 / \sqrt{2} \pi q_{p}$. This is in 


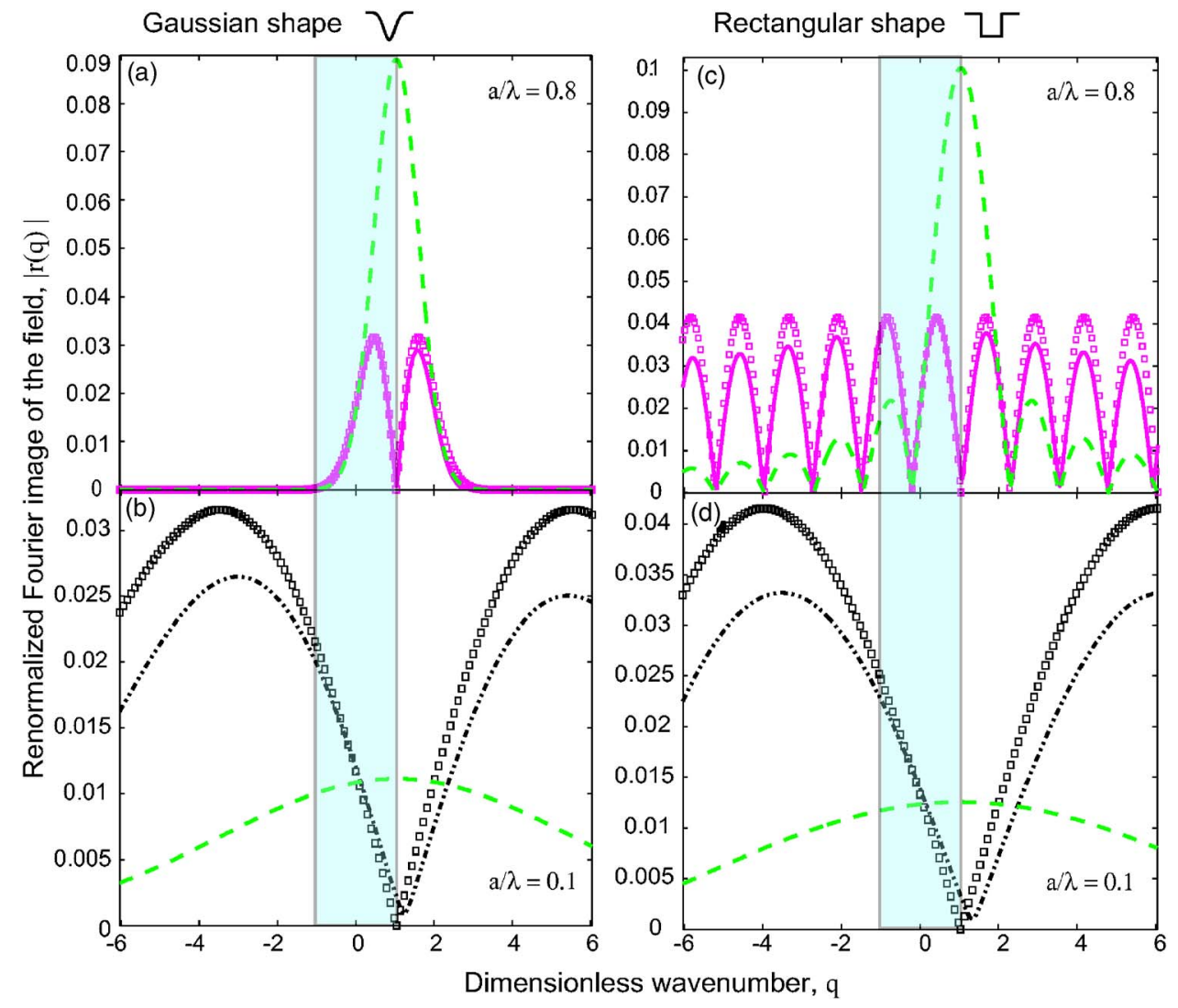

FIG. 4. (Color online) Dependence of the modulus of the renormalized Fourier image of the scattered field, $|r(q)|$, upon dimensionless wave vector $q$. Left panels show the calculation for Gaussian indentations $[a / \lambda=0.8$ in panel (a), $a / \lambda=0.1$ in panel (b)], while right panels correspond to rectangular indentations $[a / \lambda=0.8$ in panel (c), $a / \lambda=0.1$ in panel (d)]. The depth of the indentation is $w / \lambda=0.02$. In each panel the squares represent the $|r(q)|$ calculated within the first-order Born approximation, whereas dash-double-dotted (black) and solid (magenta) lines stand for the strict numerical solution; the green dashed curve renders the modulus of the $q_{p}$-shifted Fourier transformation of the defect, $\left|\eta\left(q-q_{p}\right)\right|$. Notice the dips of all curves at $q=q_{p}$ due to the presence in the potential of the "cutting" function $\left|q-q_{p}\right|$. The blue shaded areas denote the regions in $q$ corresponding to out-of-plane radiative modes.

excellent accordance with the strict numerical solution of Eq. (9); see Figs. 2(a) and 2(b). However, as the transmittance in the FOBA does not depend upon the width of the defect, higher-order terms in the Born series are required in order to reproduce this dependence appropriately.

\section{B. Out-of-plane radiation due to a single defect}

In this section we analyze the angular distribution of the the energy radiated out of the plane after scattering. This behavior is represented in Fig. 3, which renders the radiation diagrams for SPP scattering by Gaussian indentations [panel (a)] and rectangular indentations [panel (b)], for different defect widths. The surface impedance is that of silver at $\lambda$ $=600 \mathrm{~nm}(\xi=-0.277 i)$. The case of an impedance defect (with zero impedance, as for perfect conductors) is also shown in Fig. 3(c). For comparison, the calculations for rectangular indentations were also performed with the modal expansion method [circles, Fig. 3(b)]. Again, the agreement between the two methods is quite remarkable. Figure 3 shows that the radiation diagrams present a nontrivial dependence on defect shape. For impedance defects, the angle at which maximum out-of-plane radiation occurs always points along the direction of propagation of the incident SPP. However, for narrow Gaussian relief defects $(a<\lambda / 2)$, the maximum in DRC occurs at negative angles. In this case, as $a$ increases, the angle of maximal radiation shifts from negative angles to positive ones. This behavior of Gaussian defects has also been reported for surface plasmons in a thin metal film, excited in a Kretschmman configuration. ${ }^{37}$ For rectangular defects, the DRC behavior is more complex. At small $a / \lambda$ the DRC presents one emission lobe at a negative angle; as $a$ increases, the emission lobe moves to the normal direction and after a transition point (at approximately $a / \lambda$ $=1 / 2$ ) the main lobe moves to positive angles, while a second emission lobe appears at a negative angle. Finally, for $a / \lambda \approx 1$ the amplitudes of the two lobes are comparable; see Fig. 3.

Within the FOBA, the DRC can be analytically computed. From Eqs. (19) and (17) we obtain

$$
D_{1}(\theta)=\frac{4 \pi q_{p}|\xi|\left(\sin \theta-q_{p}\right)^{2} \cos ^{2} \theta}{|\xi|^{2}+\cos ^{2} \theta}\left|\eta\left(\sin \theta-q_{p}\right)\right|^{2} .
$$

As illustrated in Fig. 3(a) for the case of Gaussian indentations, FOBA results (square symbols) provide an excellent 


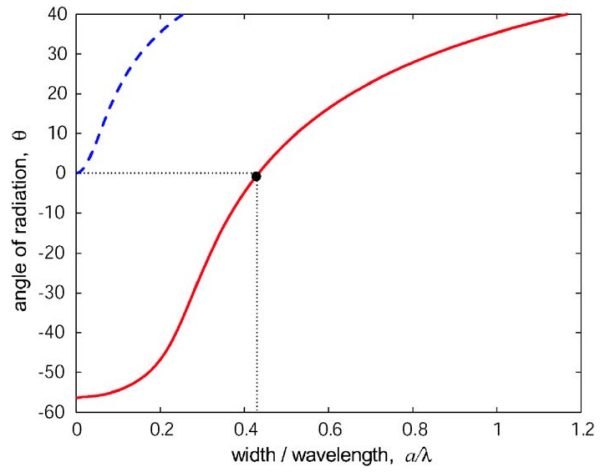

FIG. 5. (Color online) Dependence of the angle of maximum out-of-plane radiation as a function of $a / \lambda$ for Gaussian defects. The case of relief defects is represented as the red solid curve; the blue dashed curve corresponds to impedance defects.

approximation to the full solution. This allows us to connect the DRC with the potentials corresponding to the SPP scattering within such approximation.

For the case of impedance defects, Eqs. (13) and (9) imply that the renormalized spectrum of the field $r(q)$ is proportional to $\eta\left(q-q_{p}\right)$, which is the Fourier image of the defect shifted by the SPP wave vector. ${ }^{39}$ Conversely, for relief defects, $r(q)$ is proportional not only to $\eta\left(q-q_{p}\right)$ but also to $q-q_{p}$. This is illustrated in Fig. 4, for Gaussian (left panels) and rectangular (right panels) at $a / \lambda=0.8$ (top panels) and $a / \lambda=0.1$ (bottom panels). In all panels the modulus of the shifted Fourier image of the defect $\left|\eta\left(q-q_{p}\right)\right|$ is represented by the green dashed lines. Figure 4 also shows the corresponding $|r(q)|$ for relief defects, obtained by solving the integral equation (9) either strictly or within the FOBA.

As expected, the narrower the defect, the more extended $\left|\eta\left(q-q_{p}\right)\right|$ [and therefore $\left.|r(q)|\right]$ in $q$ space. It is also clear that $\left|\eta\left(q-q_{p}\right)\right|$ is a smooth function for Gaussian defects, while it presents oscillations for rectangular ones. These two properties are extremely useful in order to understand the behavior of DRC. Notice that $D(\theta)$ essentially follows the dependence of $|r(q)|^{2}$ [see Eq. (17) within the range $-1<q$ $<1$-i.e., the region corresponding to radiative modes, represented by the blue shaded areas in Fig. 4]. For impedance defects $\left|\eta\left(q-q_{p}\right)\right|$ presents its maximum at $q>0$, so the maximum out-of-plane emission always occurs in the forward direction, exactly in the same way as in the model of the local surface impedance approximation. ${ }^{31-33}$ However, for relief defects, forward-direction emission is further inhibited by the presence of the "cutting function" $q-q_{p}$ in the expression for $r(q)$. For narrow defects this results in $|r(q)|$ 's having more weight in the $q<0$ region, so the emission is directed backwards. For wide Gaussian defects $(a>0.5 \lambda)$ the width in $q$ space of $|\eta(q)|$ is smaller than $q_{p}$ (recall that $q_{p} \approx 1$ ) and the emission occurs in the forward direction. For rectangular defects, as the defect widens, more and more oscillations in $\left|\eta\left(q-q_{p}\right)\right|$ enter into the $-1<q<1$ region, leading to different emission lobes, as shown in Fig. 3(b).

The angles of maximum out-of-plane emission can be obtained in the FOBA by solving $\partial_{\theta} D_{1}(\theta)=0$. While in the case of a rectangular shape this equation is transcendent with respect to variables $\theta$ and $a / \lambda$, it may be solved explicitly for Gaussian shapes. Thus, we find that the maxima condition for Gaussian reliefs is

$$
\frac{a}{\lambda}(\theta)=\sqrt{\frac{2}{\pi^{2}\left(\sin \theta-q_{p}\right)^{2}}+\varphi(\theta)},
$$

where

$$
\varphi(\theta)=\frac{2|\xi|^{2} \tan \theta}{\pi^{2}\left(|\xi|^{2}+\cos ^{2} \theta\right)\left(q_{p}-\sin \theta\right) \cos \theta},
$$

while in the case of an impedance defect this condition is much simpler, $a / \lambda(\theta)=\sqrt{\varphi(\theta)}$. These dependences are presented in Fig. 5, reflecting the fact that while impedance defects emit preferentially in the forward direction, emission maxima occur in the backward direction up to $a / \lambda \approx 0.42$ for the relief case.

\section{CONCLUSIONS}

We have developed an approximate method (based on Rayleigh expansion and surface impedance boundary conditions) for the study of SPP scattering at arbitrary 1D inhomogeneities and applied it to the case of single defects.

We have compared the numerical solution of the integral equation in $k$ space with the calculations made by using a mode expansion technique. The excellent agreement between them (together with the fulfillment of the energy conservation law) indicates that the theoretical formulation is correct and much more accurate than previous approximations based on local surface impedances.

The case of a single-scattering center is analyzed in detail. We have compared the scattering by impedance and relief inhomogeneities of different shapes, considering both protrusions and indentations. We have shown that the transmission, reflection, and out-of-plane scattering are defined essentially by the spectral properties of the inhomogeneity. We have shown that out-of-plane radiation after scattering by impedance inhomogeneities is always directed in the forward direction (with respect to the SPP propagation). On the contrary, in the case of relief defects, the radiated energy may be directed into backward or forward directions (or both, in the case of rectangular defects), depending on the defect width and shape.

Further theoretical work will be aimed at the scattering of SPP by the multiple scatterers and, also, at the generalization of this approach to $2 \mathrm{D}$ inhomogeneities.

\section{ACKNOWLEDGMENTS}

We are grateful to A. V. Kats and J. A. Sánchez-Gil for helpful discussions and criticism. The authors acknowledge financial support from the European Network of Excellence Plasmo-Nano-Devices (Grant No. FP6-2002-IST-1-507879) and the STREP "Surface Plasmon Photonics" (Grant No. FP6-NMP4-CT2003-505699). 
*Electronic address: alexeynik@rambler.ru

†Electronic address: 1mm@unizar.es

${ }^{1}$ W. L. Barnes, A. Dereux, and T. W. Ebbesen, Nature (London) 424, 824 (2003).

${ }^{2}$ S. A. Maier, Curr. Nanosci. 1, 17 (2005).

${ }^{3}$ M. C. Hutley and D. Maystre, Opt. Commun. 19, 431 (1976).

${ }^{4}$ A. V. Kats and I. S. Spevak, Phys. Rev. B 65, 195406 (2002).

${ }^{5}$ G. P. Bryan-Brown, M. S. Hutley, and I. R. Sambles, J. Mod. Opt. 37, 1227 (1990).

${ }^{6}$ A. V. Kats, M. L. Nesterov, and A. Yu. Nikitin, Phys. Rev. B 72, 193405(B) (2005).

${ }^{7}$ T. W. Ebbesen, H. J. Lezec, H. F. Ghaemi, T. Tio, and P. A. Wolff, Nature (London) 391, 667 (1998).

${ }^{8}$ L. Martín-Moreno, F. J. García-Vidal, H. J. Lezec, K. M. Pellerin, T. Thio, J. B. Pendry, and T. W. Ebbesen, Phys. Rev. Lett. 86, 1114 (2001).

${ }^{9}$ S. C. Kitson, W. L. Barnes, and J. R. Sambles, Phys. Rev. Lett. 77, 2670 (1996).

${ }^{10}$ S. I. Bozhevolnyi, J. Erland, K. Leosson, P. M. W. Skovgaard, and J. M. Hvam, Phys. Rev. Lett. 86, 3008 (2001).

${ }^{11}$ M. Kretschmann and A. A. Maradudin, Phys. Rev. B 66, 245408 (2002).

${ }^{12}$ A. V. Kats and A. Y. Nikitin, Proc. SPIE 5221, 218 (2003).

${ }^{13}$ M. U. González, J.-C. Weeber, A.-L. Baudrion, A. Dereux, A. L. Stepanov, J. R. Krenn, E. Devaux, and T. W. Ebbesen, Phys. Rev. B 72, 161405 (2006).

${ }^{14}$ J.-C. Weeber, Y. Lacroute, A. Dereux, E. Devaux, T. Ebbesen, C. Girard, M. U. González, and A.-L. Baudrion, Phys. Rev. B 70, 235406 (2004).

${ }^{15}$ J. R. Krenn, H. Ditlbacher, G. Schider, A. Hohenau, A. Leitner, and F. R. Aussenegg, J. Microsc. 209, 167 (2003).

${ }^{16}$ J.-C. Weeber, J. R. Krenn, A. Dereux, B. Lamprecht, Y. Lacroute, and J. P. Goudonnet, Phys. Rev. B 64, 045411 (2001).

${ }^{17}$ S. I. Bozhevolnyi, V. S. Volkov, E. Devaux, J.-J. Laluet, and T. W. Ebbesen, Nature (London) 440, 508 (2006).

${ }^{18}$ F. Pincemin, A. A. Maradudin, A. D. Boardman, and J.-J. Greffet, Phys. Rev. B 50, 15261 (1994).

${ }^{19}$ O. J. F. Martin, C. Girard, and A. Dereux, Phys. Rev. Lett. 74, 526 (1995).
${ }^{20}$ T. Søndergaard and S. I. Bozhevolnyi, Phys. Rev. B 67, 165405 (2003); 69, 045422 (2004); 71, 125429 (2005).

${ }^{21}$ B. T. Draine, Astrophys. J. 333, 848 (1988).

${ }^{22}$ A. B. Evlyukhin, S. I. Bozhevolnyi, A. L. Stepanov, and J. R. Krenn, Appl. Phys. B: Lasers Opt. 84, 29 (2006).

${ }^{23}$ A. B. Evlyukhin and S. I. Bozhevolnyi, Laser Phys. Lett. 3, 396 (2006).

${ }^{24}$ F. J. García-Vidal, H. J. Lezec, T. W. Ebbesen, and L. MartinMoreno, Phys. Rev. Lett. 90, 213901 (2003).

${ }^{25}$ J. Bravo-Abad, F. J. García-Vidal, and L. Martin-Moreno, Phys. Rev. Lett. 93, 227401 (2004).

${ }^{26}$ F. López-Tejeira, F. J. García-Vidal, and L. Martín-Moreno, Phys. Rev. B 72, 161405(R) (2005).

${ }^{27}$ A. V. Zayats, I. I. Smolyaninov, and A. A. Maradudin, Phys. Rep. 408, 131 (2005).

${ }^{28}$ F. Toigo, A. Marvin, V. Celli, and N. R. Hill, Phys. Rev. B 15, 5618 (1977).

${ }^{29}$ V. I. Tatarskii, J. Opt. Soc. Am. A 12, 1254 (1995).

${ }^{30}$ A. V. Shchegrov, I. V. Novikov, and A. A. Maradudin, Phys. Rev. Lett. 78, 4269 (1997).

${ }^{31}$ A. A. Maradudin, in Topics in Condensed Matter Physics, edited by M. P. Das (Nova, New York, 1994).

${ }^{32}$ J. A. Sánchez-Gil, Appl. Phys. Lett. 73, 3509 (1998).

${ }^{33}$ J. A. Sánchez-Gil and A. A. Maradudin, Phys. Rev. B 60, 8359 (1999).

${ }^{34}$ L. Tsang, J. A. Kong, and K.-H. Ding, Scattering of Electromagnetic Waves (Wiley, New York, 2000).

${ }^{35}$ L. D. Landau, E. M. Lifshitz, and L. P. Pitaievskii, Electrodynamics of Continuous Media, 2nd ed. (Pergamon Press, New York, 1984).

${ }^{36}$ Everywhere we take into account that, due to absence of dissipation, $G\left(q_{p}\right)^{-1}=0$.

${ }^{37}$ J. Gaspar-Armenta, R. García-Llamas, and J. Durán-Favela, Phys. Rev. B 73, 155412 (2006).

${ }^{38}$ Recall though that $\eta(q)$ must be a small parameter: even for small defect depths the approximation breaks down for very stretched defects.

${ }^{39}$ Evidently, the true spectrum of the field, $H_{y}(k)$, is also strongly influenced by the SPP Green's function; see Eq. (8). 